cerimonie di danze. Già allora esistevano nella regione cinque missioni cattoliche e una protestante, inoltre un campo di polizia con prigionieri indonesi. Questi avamposti diedero inizio a una profonda metamorfosi della cultura delle popolazioni indigene. Ritornato a Giava, Helm inoltrò una mozione alla residenza olandese delle Molucche affinchè venisse creata una riserva nell'est di Kugapa allo scopo di salvaguardare le caratteristiche culturali di quegli indigeni. Purtroppo il lago Paniai era già stato prescelto per la costruzionè di un aeroporto militare e poco dopo scoppiò la seconda guerra mondiale che rese vana ogni iniziativa.

\title{
DIE VARIABILITÄT, GEOGRAPHISCHE VERTEILUNG UND STELLUNG DER KÖRPERGRÖSSE DER EINGEBORENEN NEUIRLANDS
}

\section{Отto Schlaginhaufen}

Mit 4 Abbildungen

Innerhalb der Inselflur des Pazifischen Ozeans nimmt Melanesien das Südwestgebiet ein. Seine Glieder sind durchschnittlich massiger als diejenigen Mikronesiens und Polynesiens, wenn wir von dem aus dem allgemeinen Inselfeld stark nach Süden vorstoßenden Neu-Seeland absehen. Melanesien hebt sich aber vor allem durch die Natur seiner Bevölkerung heraus, die durch dunkle Haut, krauses (helicotriches) Haar und mehr oder weniger stark ausgesprochene Prognathie charakterisiert ist. $\mathrm{Da} \beta$ man in manchen Randgebieten, wie z. B. auf den Loyalty-Inseln, Übergangstypen $\mathrm{zu}$ den mikro-polynesischen Menschenformen begegnet, ist einleuchtend. Im allgemeinen treten zwischen den einzelnen Stämmen resp. Bevölkerungsteilen die übereinstimmenden und verbindenden Merkmale und Merkmalskomplexe stärker hervor als die trennenden, soda $\beta$ uns die Bevölkerung Melanesiens trotz ihrer - stellenweise komplizierten - Gliederung in somatische Untergruppen als Einheit erscheint.

Es fehit uns aber noch vieles, um uns ein vollständiges Bild von den gegenwärtigen anthropologischen Verhältnissen Melanesiens oder gar von der Geschichte ihres Zustandekommens zu machen, und es ist daher notwendig, daß die bis jetzt vorliegenden Beobachtungsergebnisse, Merkmal für Merkmal, durchgangen und bearbeitet werden. Während eines mehr als zweijährigen Aufenthaltes (1907-1909) in Melanesien hatte ich Gelegenheit, in verschiedenen Gegenden dieser Südseeregion, namentlich auf Netirland (Neumecklenburg), einer der großen Inseln des Bismarck-Archipels, neben meinen ethnographischen Arbeiten anthropologische Untersuchungen vorzunehmen. Ihre Resultate dürften einen erwünschten Beitrag zur Kenntnis des somatischen Verhaltens der Bevölkerung Melanesiens darstellen. Wenn im folgenden speziell die Körpergröße einer Betrachtung unterzogen werden soll, so einmal deswegen, weil dieses Merkmal sowohl von anderen Autoren als auch von mir selbst in solcher Häufigkeit untersucht worden ist, daß sich ausreichende Grundlagen für die Kenntnis seiner Variabilität und geographischen Verbreitung ergeben haben, dann aber auch deshalb, weil das Problem des rassenmäßigen Kleinwuchses, die sogenannte Pygmäenfrage, in das melanesische Gebiet übergreift.

Bei meinen eigenen Untersuchungen, über die hier berichtet werden soll, wurden Rudolf MARTiNs Methoden und Instrumente angewendet. Die Bestimmung der Körpergröße ist wohl noch in höherem Maße als diejenige anderer körperlicher Merkmale den wechselnden Bedingungen ausge setzt, wie sie sich auf einer Forschungsreise bieten und die genaue Befolgung der methodischen Vorschriften erschweren. Doch war es mir möglich, die Körpergröße an 1007 erwachsenen Eingeborenen Neuirlands und der ihm vorgelagerten Inseln festzustellen.

Der Durchschnitt, der sich für 946 Männer ergab, beträgt $1610 \pm 2.0 \mathrm{~mm}$; die Individualzahlen schwanken zwischen 1350 und $1803 \mathrm{~mm}$. Im weiblichen Geschlecht liegt für 61 Personen der Mittelwert bei $1502 \pm 5.6 \mathrm{~mm}$, das Minimum bei 1396 und das Maximum bei $1618 \mathrm{~mm}$. Beide Mittelwerte fallen in die Kategorie «Untermittelgroß» von Martins (1928, 246) Einteilung, und der weibliche macht $93.3 \%$ des männlichen aus. Wie die Einzelfälle sich in die Kategorien einordnen, geht aus Tabelle 1 hervor. Bei den Männern sind die Kleinen am stärksten, die Untermittelgroßen am zweitstärksten und die Mittelgroßen am dritt- 
stärksten vertreten, während bei den Frauen die Untermittelgroßen an erster, die Kleinen an zweiter und die Mittelgroßen an dritter Stelle stehen.

Tabelle 1

Verteilung auf die Kategorien der Körpergröße

\begin{tabular}{|c|c|c|c|c|c|c|}
\hline \multirow{2}{*}{$\begin{array}{l}\text { Bezeichnung der } \\
\text { Kategorien }\end{array}$} & \multicolumn{3}{|c|}{$\overrightarrow{0}$} & \multicolumn{3}{|c|}{ q } \\
\hline & Abgrenzung & abs. & $\%$ & Abgrenzung & abs. & $\%$ \\
\hline $\begin{array}{l}\text { Zwergwuchs } \\
\text { Sehr klein } \\
\text { Klein }\end{array}$ & $\begin{array}{r}\text { bis } 129,9 \\
130,0-149,9 \\
150,0-159,9\end{array}$ & $\begin{array}{r}\overline{29} \\
375\end{array}$ & $\begin{array}{r}\overline{3,04} \\
39,64\end{array}$ & $\begin{array}{r}\text { bis } 120,9 \\
121,0-139,9 \\
140,0-148,9\end{array}$ & $\begin{array}{r}- \\
21\end{array}$ & $\begin{array}{r}\overline{1.64} \\
34,43\end{array}$ \\
\hline $\begin{array}{l}\text { Untermittelgroß } \\
\text { Mittelgroß } \\
\text { Übermittelgroß }\end{array}$ & $\begin{array}{l}160,0-163,9 \\
164,0-166,9 \\
167,0-169,9\end{array}$ & $\begin{array}{r}258 \\
126 \\
92\end{array}$ & $\begin{array}{r}27,28 \\
13,32 \\
9,74\end{array}$ & $\begin{array}{l}149,0-152,9 \\
153,0-155,9 \\
156,0-158,9\end{array}$ & $\begin{array}{r}25 \\
10 \\
1\end{array}$ & $\begin{array}{r}40,97 \\
16,40 \\
1,64\end{array}$ \\
\hline $\begin{array}{l}\text { Groß } \\
\text { Sehr groß } \\
\text { Riesenwuchs }\end{array}$ & $\begin{array}{c}170,0-179,9 \\
180,0-199,9 \\
200,0 \text { u. darübe }\end{array}$ & $\begin{array}{r}65 \\
1 \\
-\end{array}$ & $\begin{array}{l}6,88 \\
0,10 \\
-\end{array}$ & $\begin{array}{l}159,0-167,9 \\
168,0-186,9 \\
187,0 \text { u. darübe }\end{array}$ & $\frac{3}{-}$ & $\frac{4,92}{-}$ \\
\hline
\end{tabular}

$\mathrm{Daß}$ sich die Körpergröße der Neuirländer beträchtlich unter derjenigen mitteleuropäischer Gruppen hält, mag ein Vergleich mit der schweizerischen Bevölkerung zeigen.

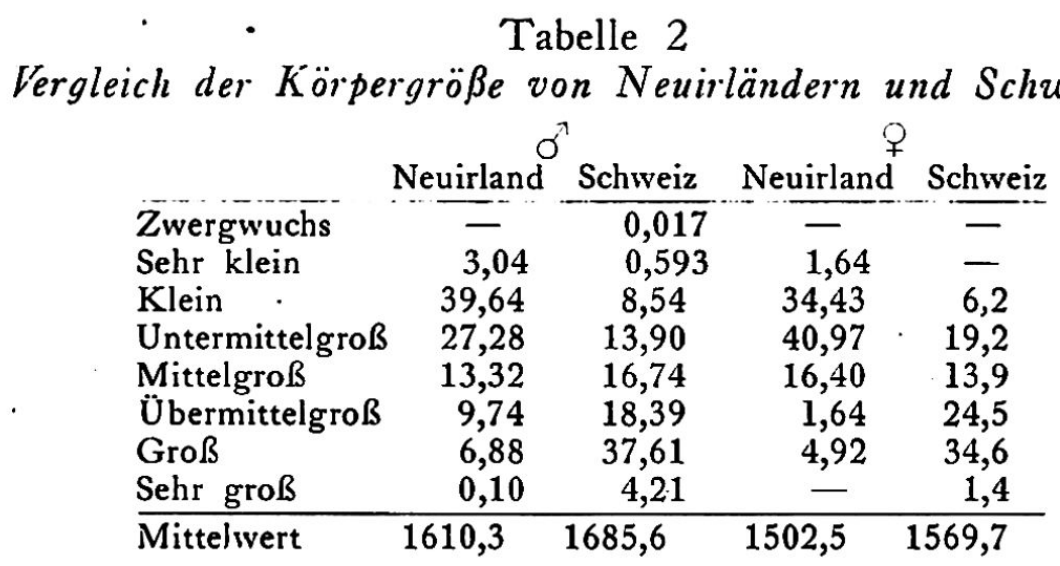

Die Körpergrößen der Schweizer Männer, die den Ergebnissen dcr anthropologischen Untersuchungen an den schweizerischen Stellungspflichtigen der Jahre 1927-1932 entnommen sind (Schlaginhaufen 1946, 51), besetzen die Kategorie „Groß“ am stärksten; es folgen die Übermittelgroßen, die nur halb soviele Fälle auf sich vereinigen, und dann die Mittelgroßen. Für den Vergleich mit den Neuirländerinnen wählte ich die Frutigtalerinnen (BossharT 1938, 19), die eher zu kleinerem Wuchs neigen, aber gleichwohl mit einem Drittel der Fälle der Kategorie „Groß“ und mit einem Viertel der Kategorie "Übermittelgroß" angehören; die Untermittelgroßen folgen an dritter Stelle mit gegen einem Fünftel der Individuen, während diese Kategorie bei den Neuirländerinnen die am stärksten frequentierte ist.

Ein Blick auf die männliche Variationskurve der Neuirländer (Fig. 1) läßt ihre Aufspaltung in zwei Gipfel erkennen, deren größerer seine Spitze bei $161 \mathrm{~cm}$, deren kleinerer sie bei $157 \mathrm{~cm}$ hat. Davon abgesehen erscheint das Polygon weitgehend symmetrisch.

Der Kartenskizze (Fig. 2) ist zu entnehmen, wo die Wohnsitze der 23 verschiedenen und hier mit den Buchstaben $a-x$ bezeichneten. Menschengruppen liegen, von denen ich Eingeborene gemessen habe.' Es entfallen acht Gruppen auf NordNeuirland und je fünf auf Mittel-Neuirland, Süd-Neuirland und die vorgelagerten Inseln. Dazu kommt noch die westlich von der Südspitze wohnende Gruppe Kambotoros-Lamassa, die von Friedericr (1912) gemessen wurde. Das Kartenbild in Verbindung mit Tabelle 3 gibt uns Aufschluß über die Körpergröße der einzelnen Gruppen und über deren geographische Verteilung. Tabelle 3 enthält auch die statistischen Kœffizienten, nämlich $M=$ arithmetischer Mittelwert, $V_{1}$ $=$ individuelles Minimum, $\mathrm{Vn}=$ individuelles Maximum, $\varepsilon=$ durchschnittliche Abweichung, $\sigma=$ standard deviation, $w$ und $\mathrm{v}=$ Variationskœffizienten, $\mathrm{m}=$ mittlerer Fehler des arithmetischen Mittels. 


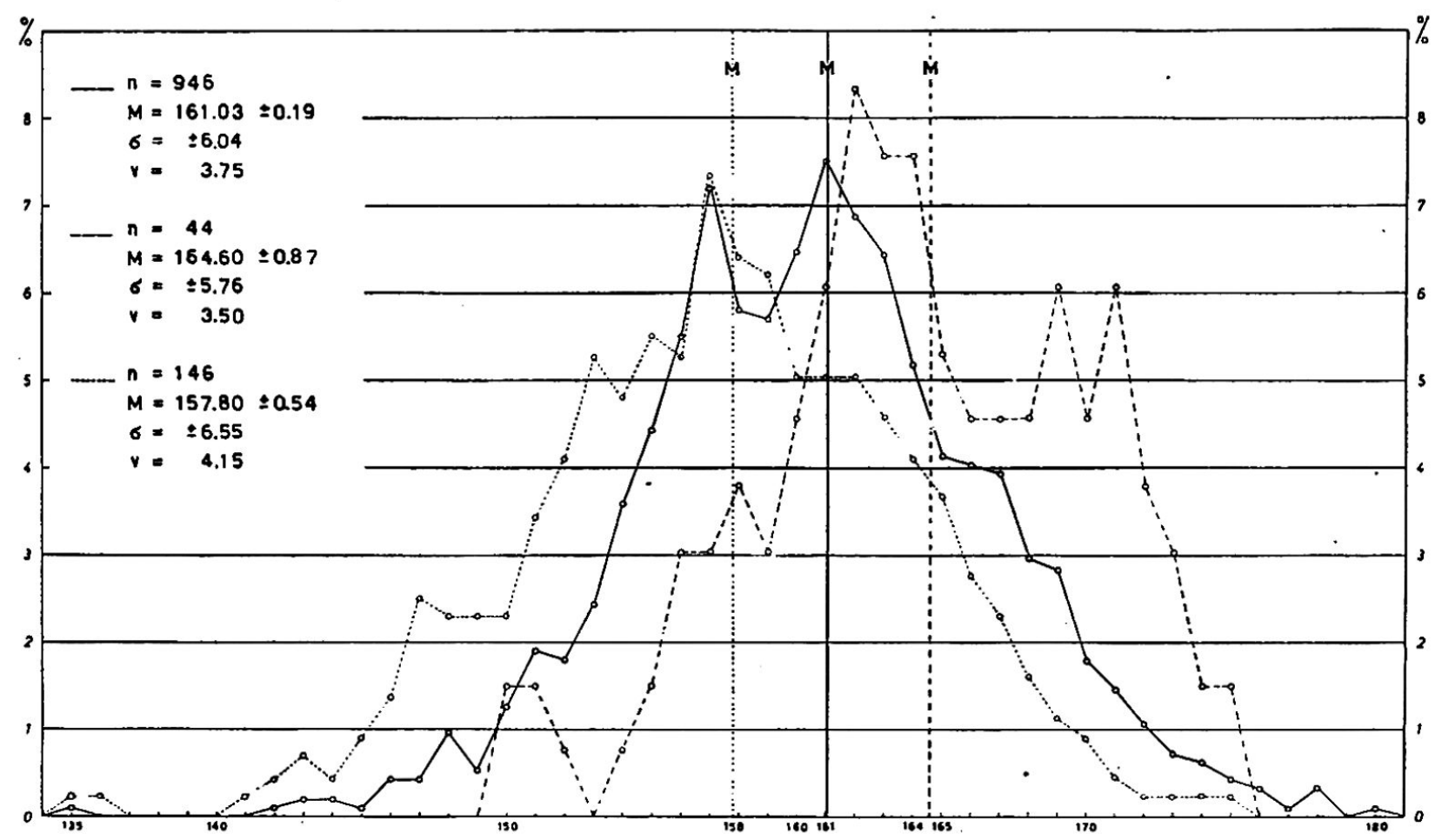

Fig. 1 Frequenzkurven der Körpergröße prozentual dargestellt

- Gesamtgruppe; …... Gruppe v, Insel Lir; -...- Gruppe g, Panegendu in Nord-Neuirland

Die Durchschnittszahlen der 23 Gruppen, aus denen sich das gesamte neuirländische Material zusammensetzt, steigen im männlichen Geschlecht von 1551.0 bis $1655.0 \mathrm{~mm}$ auf. Ihrer 14 sind als untermittelgro $\beta$, ihrer sechs als klein und ihrer drei als mittelgroß zu bezeichnen. Bei den acht weiblichen Gruppen wurden Mittelwerte von 1475.0 bis $1553.5 \mathrm{~mm}$ berechnet. Fünf Mittelwerte entfallen auf die untermittelgroßen, zwei auf die kleinen und èiner auf die mittelgroßen Körperlängen.

Zieht man die geographisch benachbarten Gruppen zu größeren Gruppen zusammen, so sieht man, daß auf der Hauptinsel, deren Mittelwert $1615.3 \pm 2.1 \mathrm{~mm}$ beträgt, die durchschnittliche Körpergröße von Norden nach Süden etwas abnimmt. Im Nordteil beläuft sie sich auf $1616.4 \pm 2.8 \mathrm{~mm}$, im mittleren auf $1614.1 \pm$ $5.1 \mathrm{~mm}$ und $\mathrm{im}$ südlichen auf $1613.8 \pm 4.4 \mathrm{~mm}$. Doch lassen die wahrscheinlichen Fehler erkennen, daß die Differenzen zwischen den drei Gruppen nicht ins Gewicht fallen. Schärfer markiert sich der Unterschied zwischen der Hauptinsel und den vorgelagerten Inseln. Die letzteren weisen einen Mittelwert von $1596.6 \pm 4.3 \mathrm{~mm}$ auf und stehen somit um $18.7 \mathrm{~mm}$ hinter der Hauptinsel zurück. Indessen sind nicht alle Inselgruppen an dieser Herabminderung ihres Gesamtdurchschnitts beteiligt. Die Tangainseln $(w)$ zeichnen sich mit $1645.4 \pm 10.2 \mathrm{~mm}$ sogar durch eine Statur aus, die sie die dritte Stelle unter den 23 Einzelgruppen einnehmen läßt, während Lir (v) mit $1578.0 \pm 5.4 \mathrm{~mm}$ die zweitniedrigste Körpergröße besitzt. $\mathrm{Da}$ Lir die umfangreichste der gemessenen Einzelgruppen ist, trägt sie die Hauptverantwortung für den in die Spitze 157 auslaufenden Nebengipfel der Frequenzkurve des Gesamtmaterials, den wir früher schon erwähnten (Fig. 1). Es sollen nun die Inseln nicht nur mit der Hauptinsel als Ganzes verglichen, sondern auch eine jede zu dem ihr gegenüberliegenden Küstenstrich der Hauptinsel in Vergleich gesetzt werden. Bei früherer Gelegenheit (Schlagin Haufen 1910) habe ich, entsprechend der Linie Tanga - Muliama - Butam einen Querschnitt durch den Südteil unseres Gebietes geführt und hinsichtlich der Körpergröße festgestellt, daß sie auf Tanga am größten, an der Küste von Muliama kleiner und in den. hinter Muliama aufsteigenden Bergen der Butam am kleinsten ist. Die entsprechenden männlichen Mittelwerte sind: Tanga (w) $1645.4 \pm 10.2 \mathrm{~mm}$, Muliama (q) 1613.7 
Tabelle 3

Die Körpergrößc neuirländischen Menschengruppen und ihre statistischen Koefficienten

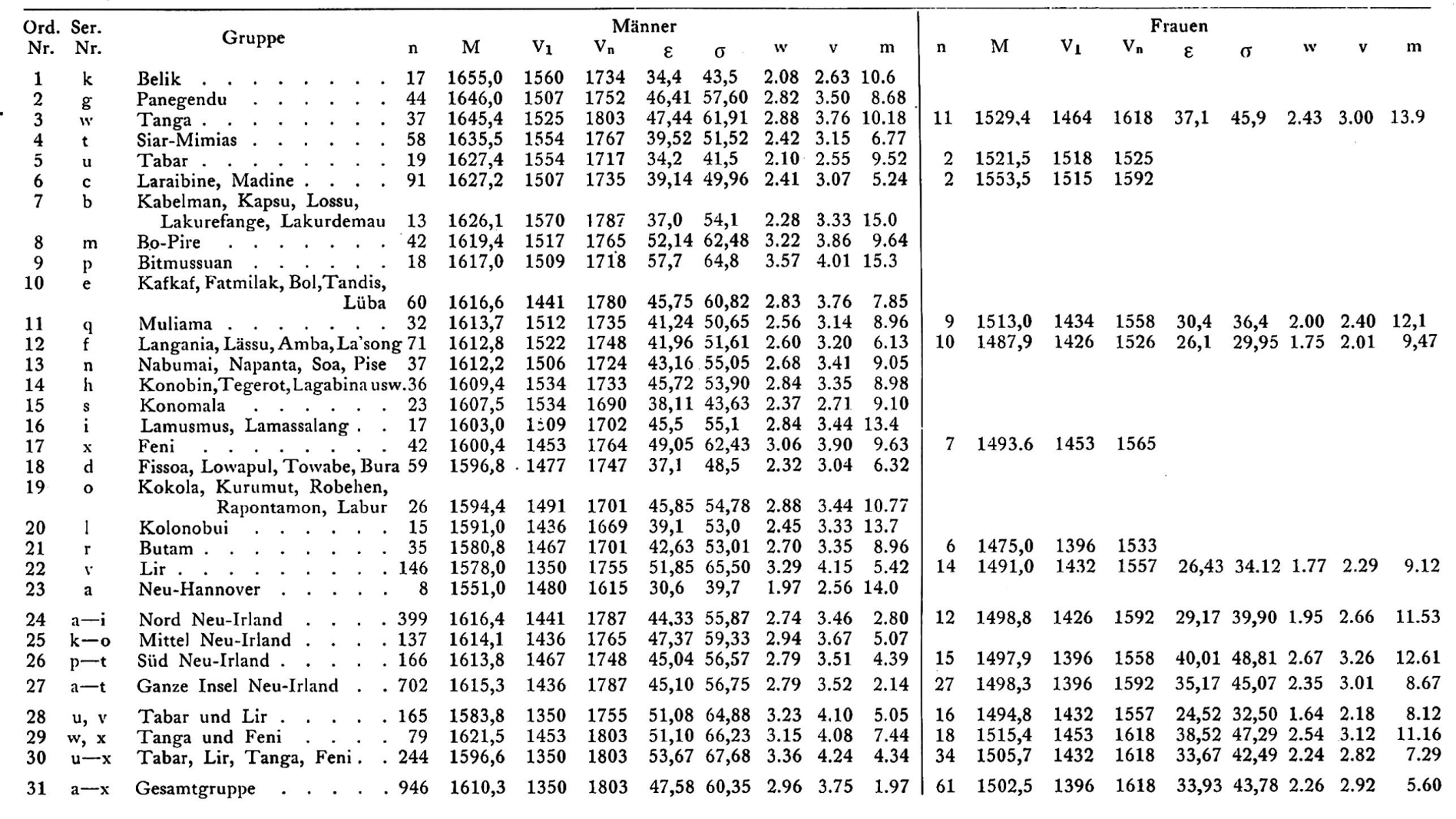


$\pm 8.9 \mathrm{~mm}$, Butam (r) $1580.8 \pm 8.9 \mathrm{~mm}$. Führt man einen solchen Querschnitt von den Feni-Inseln ( $x$ ) her, so findet man, daß auf diesen die mittlere Körperlänge sich nicht einmal ganz in der Höhe der gegenüber liegenden Küste hält. Sie beträgt $1600.4 \pm 9.6 \mathrm{~mm}$, während Muliama $1613.7 \pm 8.9 \mathrm{~mm}$ und die südlich daran anschließende Küstenlandschaft Konomala (s) $1607.5 \pm 9.1 \mathrm{~mm}$ aufweisen. Von Lir aus läßt sich die Linie durch die Küstenlandschaft von Belik (k) oder diejenige von Kolonobui (l) ziehen. Im ersteren Fall verbindet sie die beiden Gruppen mit den extremsten von mir festgestellten Durchschnittswerten unseres Gebietes: Lir $1578.0 \pm 5.4 \mathrm{~mm}$, Belik $1655.0 \pm 10.6 \mathrm{~mm}$. Im letzteren Fall dagegen führt der Schnitt durch zwei Gruppen, die sich in der Körpergröße von Lir nur wenig unterscheiden: Kolonobui mit $1591.0 \pm 13.7 \mathrm{~mm}$ und die West-Küstenstrecke Kokola - Labur (0) mit $1594.4 \pm 10.8 \mathrm{~mm}$. Eher im Sinne des Tangaquerschnitts liegen die Verhältnisse auf der von der Insel Tabar (u) ausgehenden Linie. Auf Tabar beträgt die männliche Körpergröße $1627.4 \pm 9.52 \mathrm{~mm}$, in Fissoa (d) und Nachbarlandschaften $1596.8 \pm 6.32 \mathrm{~mm}$ und an dem Westküstenstrich Lamusmus Lamassalang (i) $1603.0 \pm 13.4 \mathrm{~mm}$. Auf die Beziehungen zwischen Neu-Hannover (a) und dem. Nordteil der Hauptinsel einzutreten, dürfte sich kaum lohnen, da der Mittelwert der Neu-Hannoveraner von $1551.0 \pm 14.0 \mathrm{~mm}$ sich auf die Beobachtungen an nur 8 Männern gründet und daher wohl kaum repräsentativ sein kann.

Unsere Liste der Körpergrößenmittel enthält deren drei, die von Inlandstämmen herrühren: 1. von den Butam (r) $(1580.8 \pm 8.96 \mathrm{~mm}), 2$. von den Eingeborenen der mittel-neuirländischen Orte Nabumai, Napanta, Soa und Pisé (n) (1612.2 $\pm 9.05 \mathrm{~mm}), 3$. von den Eingeborenen der schon zu Nord-Neuirland zu rechnenden Orte Konobin, Tegerot, Lagabina (h) $(1609.4 \pm 8.98 \mathrm{~mm})$. Der erste der drei Durchschnittswerte reiht sich in die Kategorie der «Kleinen 》 ein, die beiden andern sind «untermittelgroß». Ich habe in einer früheren Arbeit (ScHLAGINHAUFEN 1914) darauf hingewiesen, daß auf Neu-Guinea und im Bismarck-Archipel die Körpergröße von der Küste nach dem Innern - wenige Stämme ausgenommen - abnimmt. Dies trifft für die Abschnitte Neuirlands, aus denen uns die Körpergröße von Inlandstämmen bekannt sind, zu. Im Süden heben sich die Butam durch ihre kleine Statur deutlich von den Küstenstämmen in Bitmussuan, Muliama, Konomala und Siar-Mimias (t) und noch schärfer, von der der Südspitze naheliegenden Gruppe Kambotoros-Lamassa ab, deren Mittelwert von Friederici (1912) zu $165.9 \mathrm{~cm}$ bestimmt wurde. Im Nordteil sind die Inlandstämme von Konobin, Tegerot usw. durch ihren kleinen Wuchs von den Küstenleuten in Panégendu stark, von denjenigen in Langania, Lässu usw. schwach unterschieden. Im Mittelteil ist die Stellung der Inlandleute von Nabumai, Napanta usw. gegenüber den Eingeborenen der beiden Küsten verschieden, indem sie kleiner als die Leute von Bo-Pire $(\mathrm{m})$, aber größer als die Männer von Kokola - Labur ( $\sigma$ ) sind.

Von anderen Autoren sind die folgenden Angaben über die Körpergröße von Neuirländern zu erwähnen :

HAGEN (1898) hat an 11 erwachsenen Männern aus Neuirland die Körpergröße gemessen und eine von 1480 bis $1704 \mathrm{~mm}$ reichende Zahlenreihe gefunden, aus welcher sich ein Mittel von 1619 mm berechnen läßt. Fünf der Gemessenen stammen von Nusa und zeigen Werte, die sich von 1570 bis $1704 \mathrm{~mm}$ aufreihen. Für 5 Frauen von verschiedenen Stellen Neuirlands ergaben sich Maßzahlen von 1393 bis $1578 \mathrm{~mm}$. Der Mittelwert beträgt $1514 \mathrm{~mm}$. Die drei Männer, die STEPHan in Neuirland gemessen hat, sind 1620,1630 und $1700 \mathrm{~mm}$ groß; der größte und der kleinste stammen von der Insel Lamassa, der dritte aus Mittel-Neuirland (Stephan und Grebner 1907, 195-203). Der Durchschnitt der 26 von FRIEDERICI $(1912,323)$ in Namatanai-Bo-Piré gemessenen Leute beziffert sich auf $1618 \mathrm{~mm}$ und deckt sich fast genau mit dem von mir an 42 Individuen festgestellten Mittel von $1619,4 \mathrm{~mm}$. Eine sehr erwünschte Ergänzung zu meinem Material bilden die vom selben Autor in Kamhotoros-Lamassa untersuchten 17 Männer, da ich vom Westen der Südspitze Neuirlands wohl Schädelmaterial nicht aber Beobachtungen am Lebenden besitze. FrIEDERICIs Mittelzahl beträgt $1659 \mathrm{~mm}$. Aus seinem Material sind noch die Maßzahlen von fünf aus Lambel, d.h. dem Inland Süd-Neuirlands stammenden Männern zu erwähnen, die von 1551 bis $1640 \mathrm{~mm}$ variieren und einen Mittelwert von $1602 \mathrm{~mm}$ ergeben, und eine Frau von $1537 \mathrm{~mm}$ Körpergröße. Genauere Angaben über die Herkunft dieser Lambel-Leute liegen nicht vor; doch dürfte es sich nicht um Butam handeln. 


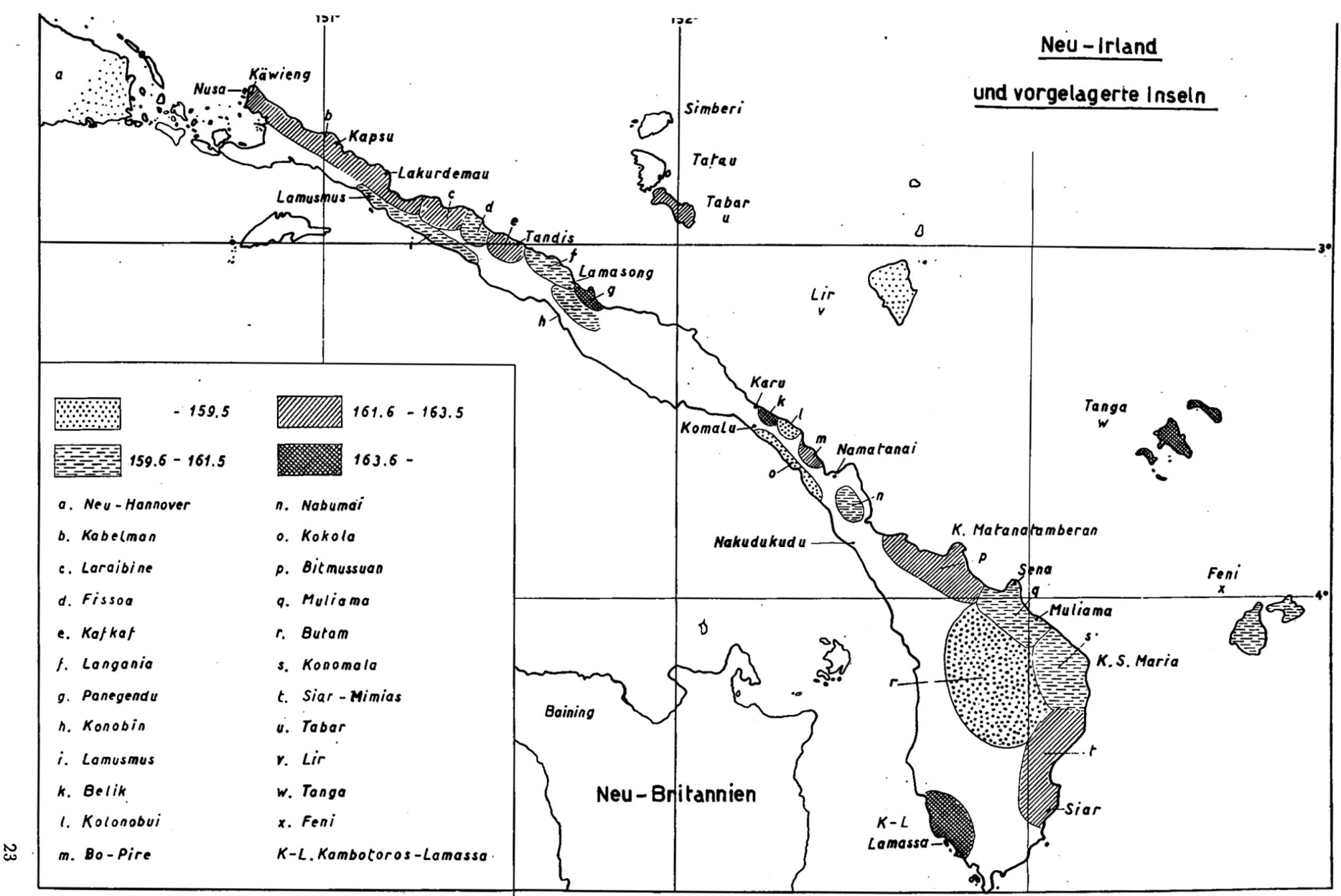

Fig. 2 Umrißkarte Neuirlands und der ihm vorgelagerten Inseln. Verteilung der Körpergröße der Eingeborenen in diesem Gebiet. Maßstab 1:2000 000 
Nach den bis heute vorliegenden Untersuchungsergebnissen darf es als feststehend bezeichnet werden, da $\beta$ auf Neuirland und den $\mathrm{ihm}$ vorgelagerten Inseln keine Menschengruppen leben, die als Pygmäen aufzufassen sind.

Um etwas über die Stellung der Neuirländer zu den Eingeborenen anderer Gebiete Melanesiens hinsichtlich der Körpergröße zu erfahren, habe ich die mir bekannten, in der Literatur figurierenden Mittelwerte melanesischer Gruppen in einer Liste zusammengestellt (Tab. 4). Man sieht, da $\beta$ diese im männlichen Geschlecht von $1384 \mathrm{~mm}$ bei den Ramu-Leuten im ehemaligen Deutsch-Neuguinea bis $1732 \mathrm{~mm}$ bei den Toaripi in Britisch - Neuguinea aufsteigen. Ob damit die Grenzen der melanesischen Durchschnittszahlen gegeben sind, steht nicht fest; denn bis jetzt ist uns nur ein Teil der Bevölkerung Melanesiens anthropologisch bekannt, und es muß zugegeben werden, daß mit der Bearbeitung bis jetzt noch nicht erforschter Gruppen die Grenzwerte eine Verschiebung und die Frequenzen der Kategorien Veränderungen erfahren können. In die Kategorien der Körpergrößeneinteilung ordnen sich die $11 \delta$ männlichen Gruppen so ein, daß 6 als sehr klein, 37 als klein, 42 als untermittelgroß, 17 als mittelgroß, 13 als übermittelgroß und 3 als groß zu bezeichnen sind. Wir wollen dabei nicht vergessen, daß die einzelnen Gruppen unter sich vielfach recht ungleichartig sich hinsichtlich Frequenz und geographischer Ausdehnung, da $\beta$ in einigen Fällen die Einzelgruppen als Bestandteile von Gesamtgruppen wiederkehren und daß die säkulare Zunahme der Körpergröße vielleicht auch in Melanesien eine Rolle spielt. Scharfe Schlüsse lassen sich daher nicht ziehen; doch besteht kaum ein $Z$ weifel, daß die Kategorien der Kleinen und Untermittelgroßen den überwiegenden Teil ausmachen, so wie sie dies auch innerhalb Neuirlands tun.

Tabelle 4

Körpergröße melanesischer Gruppen

\begin{tabular}{|c|c|c|c|c|c|c|c|c|}
\hline \multirow{2}{*}{\multicolumn{4}{|c|}{ Gruppe }} & \multicolumn{2}{|c|}{ Männer } & \multicolumn{2}{|c|}{ Frauen } & \multirow[t]{2}{*}{ Autor } \\
\hline & & & & $\mathrm{n}$ & M & & & \\
\hline Ramu, D.N.G. . &  & $\theta$ & . & 12 & 1384 & 3 & 1308 & Moyne \& Haddon (1936) \\
\hline Ramu, D.N.G. & & . & . & 20 & 1431 & & & Kirschbaum (1927) \\
\hline Tapiro, H.N.G. . & . & . & . & 22 & 1449 & & & Haddon $(1912,314)$ \\
\hline Kamaweka, B.N.G. & . & . & $\cdot$ & 11 & 1487 & & & Seligmann $(1909,329)$ \\
\hline Tapiro, H.N.G. . & & . & . & 49 & 1489 & 26 & 1383 & Bijlmer $(1939,136)$ \\
\hline Goliath, H.N.G. & & & & 12 & 1492 & & & v. d. Broek $(1913,34-36)$ \\
\hline Toricelligebirge (Aku & u. $A$ & pur) $D$ & D.N.G. & 23 & 1509 & & & Schlaginhaufen $(1914,9)$ \\
\hline Toricelligebirge (mit & Afu) & D.N. & & 30 & 1519 & & & \\
\hline Pesechem, H.N.G. & & . & . & 52 & 1524 & & & v. d. Broek $(1915,240)$ \\
\hline Kai, D.N.G. & & . & & 50 & 1525 & & & Pöch (1905 [41]) \\
\hline Pania Kapaukoes, $\mathrm{H}$. & v.G. & . & . & 300 & 1528 & 19 & 1433 & Bijlmer $(1939,137)$ \\
\hline Jera Kapaukoes, H.N & & . & & 10 & 1529 & & & $"$ \\
\hline Tiri, H.N.G. & & . & . & 20 & 1529 & & & $(1939,136)$ \\
\hline Fergusson-Inseln, B.N & G. & . & . & 11 & 1530 & & & Seligmann $(1909,270)$ \\
\hline Oeroemoeka, H.N.G. & 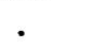 & . & . & & 1536 & & & Bijlmer $(1934,136)$ \\
\hline Geelvinkbai, H.N.G. & . & . & 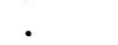 & & 1537 & & & Meyer $(1874,93)$ \\
\hline Sattelberg, D.N.G. & & . & & 273 & 1539 & 314 & 1444 & Keysser $(1911,494)$ \\
\hline Südwest-Santo, N. He & & . & . & 60 & 1545 & & & Speiser $(1928,94)$ \\
\hline Poum, D.N.G. . & . & . & . & 9 & 1547 & & & Schellong (1891, 199-201) \\
\hline Timorini, H.N.G. & . & . & . & 64 & 1547 & 20 & 1432 & Bijlmer $(1922,8-9)$ \\
\hline Mafulu, B.N.G. . & . & . & . & 20 & 1551 & & & Williamson $(1912,18)$ \\
\hline Tubetube, B.N.G. & & . & . & 10 & 1555 & & & Seligmann $(1909,271)$ \\
\hline Tumuip, N. Britannie & & . & . & 9 & 1556 & & & Friederici $(1912,322)$ \\
\hline Fakfak, H.N.G. . & & . & . & 10 & 1557 & & & $\operatorname{Koch}(1906,203-211)$ \\
\hline Mount-Hagen, B.N.G & & . & . & 53 & 1560 & 46 & 1440 & J. Lindsay Taylor (1934) \\
\hline West-Santo, N. Hebr. & & . & 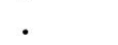 & 77 & 1568 & & & Speiser $(1928,94)$ \\
\hline Bena-Bena, B.N.G. & & . & . & 16 & 1570 & 9 & 1470 & J. Lindsay Taylor (1934) \\
\hline Baining, N. Britannie & & - & . & & 1572 & & & Thurnwald, unveröff. \\
\hline $\begin{array}{l}\text { Nassioi, Bougainville } \\
\text { Marshall Bennet-Inse }\end{array}$ & & N.G. & $\dot{.}$ & & $\begin{array}{l}1576.9 \\
1577\end{array}$ & & & $\begin{array}{l}\text { Schlaginhaufen } \\
\text { Seligmann }(1909,273)\end{array}$ \\
\hline
\end{tabular}




\section{Gruppe}

Lir (v)

Butam (r)

Koiari, B.N.G.

Jakumul, D.N.G.

Leitere, D.N.G.

Kieta, Bougainville (ganze Gruppe)

Mekeo, B.N.G.

Baining, N. Britannien

Kolonobui (l)

Espiritu Santo, Ges. gr

Kokola, Kurumut us.w. (o)

Biak, H.N.G.

Fissoa, Lowapul usw. (d)

Sentanisee, H.N.G.

Arup, D.N.G

Namau, B.N.G.

Feni (x)

Garia, B.N.G.

St. Cruz

Lamusmus, Lamassalang (i)

Biak, H.N.G.

Konomala (s)

Trobriand-Insel, B.N.G.

Jabim, D.N.G. . . . .

Konobin, Tegerot, Lagabina (h)

Neu-Irland, Gesamtgruppe .

Nabumai, Napanta, Soa us.w. (n)

Langania, Lä̈ssu usw. (f)

St. Christoval, Salomons-Inseln

Talamacco, Santo, N. Hebr. .

Muliama (q)

Oemar Kapaukoes, H.N.G. .

Jabim, D.N.G.

Daudai, B.N.G.

Kafkaf, Fatmilak us.w. (e)

Roro, B.N.G.

Neu-Mecklenburg .

Namatanai-Bo-Pire

Bo-Pire (m) .

Luf

Motu, B.N.G.

Kieta, Strandbew., Boug.

Mamberamo-Papuas, H.N.G.

Kabelman, Kapsu usiw. (b) .

Laraibine, Madine (c) .

Tabar (u)

Alu, Salomons-Inseln

Bougainville-Straits, Salomons-Ins.

Neu-Hebriden

Humboldtbai, H.N.G.

Buka, Salomons-Inseln

Siar-Mimias ( $\mathrm{t}$ )

Admiralty-Inseln .

Central-Santo, N. Heb

Lifu, Loyalty-Inseln

Etna-Bai, H.N.G.

Mimika, H.N.G.

Tanna, N. Hebr.

Tanga (w)

Mimika, H.N.G. (1931)

Panegendu (g)

Merauke, H.N.G.

\begin{tabular}{rrrr}
\multicolumn{2}{c}{ Männer } & \multicolumn{2}{c}{ Frauen } \\
n & M & n & M \\
146 & 1578 & 14 & 1491 \\
35 & 1580 & 6 & 1475 \\
10 & 1582 & & \\
100 & 1582 & & \\
21 & 1584 & & \\
34 & 1585 & & \\
29 & 1585 & & \\
78 & 1591 & &
\end{tabular}

$\begin{array}{ll}15 & 1591\end{array}$

$214 \quad 1591$

$26 \quad 1594.4$

$37 \quad 1596$

$\begin{array}{ll}59 & 1596.8\end{array}$

1597

201600

221600

$42 \quad 1600.4$

201603

$34 \quad 1603$

$\begin{array}{ll}17 & 1603.0\end{array}$

261606

$23 \quad 1607.5$

201609

$27 \quad 1609.2$

$\begin{array}{ll}36 & 1609.4\end{array}$

$31 \quad 1610$

$946 \quad 1610.3$

$37 \quad 1612.2$

$\begin{array}{ll}71 & 1612.8\end{array}$

1612.9

$22 \quad 1613$

$32 \quad 1613.7$

101614

$14 \quad 1614$

$11 \quad 1616$

$60 \quad 1616.6$

1617

$10 \quad 1617$

$18 \quad 1617.0$

$26 \quad 1618$

$42 \quad 1619.4$

$\begin{array}{ll}9 & 1621\end{array}$

$14 \quad 1621$

1624.6

$15 \quad 1625$

$13 \quad 1626.1$

$91 \quad 1627.2$

$19 \quad 1627.4$

1628.0

1631.9

$25 \quad 1632.9$

1633

11.1634

$\begin{array}{ll}58 & 1635.5\end{array}$

101636

$20 \quad 1639$

101642

$9 \quad 1643$

$14 \quad 1643$

$187 \quad 1645$

$37 \quad 1645.4$

1001646

$44 \quad 1646.0$

$46 \quad 1649$
Autor

Schlaginhaufen

Seligmann $(1909,325)$

Schlaginhaufen $(1914,25)$

$(1914,38)$

$(1914,25)$

Seligmann (1909, 321)

Friederici (1912, 322)

Schlaginhaufen

Speiser (1928, 94)

Schlaginhaufen

Bos (1935, 38)

Schlaginhaufen

v. d. Sande $(1907,345-53)$

Schlaginhaufen $(1914,35)$

Seligmann (1909, 257)

Schlaginhaufen

Seligmann (1909, 327)

Speiser (1923, 90)

Schlaginhaufen

Wirz (1925, 185-215)

Schlaginhaufen

Seligmann (1909, 273)

Schellong (1891, 194-98)

Schlaginhaufen

361460 J. Lindsay Taylor (1934)

$61 \quad 1502.5$ Schlaginhaufen

101487.9

Guppy"(1887, 103)

Speiser $(1928,94)$

$9 \quad 1513.0$ Schlaginhaufen

91473 Bijlmer (1939, 136)

Hagen $(1898,70)$

Seligmann (1909, 262)

Schlaginhaufen

Seligmann $(1909,320)$

51513 Hagen (1898, 70)

Schlaginhaufen

Friederici (1912, 323)

Schlaginhaufen

Hambruch (1909, 53)

Seligmann $(1909,316)$

Schlaginhaufen

Bijlmer (1922, 9)

Schlaginhaufen

Ribbe (1903, 334)

Guppy $(1887,103)$

Ollivier $(1951,190)$

v. d. Sande $(1907,345-53)$

Hagen $(1898,68)$

Schlaginhaufen

Speiser " $(1928,94)$

Deniker (1893)

Koch (1906, 203-211)

Humphreys (1926)

11 1529.4 Schlaginhaufen

$80 \quad 1528$ Bijlmer $(1939,136-137)$

Schlaginhaufen

Koch (1906, 203-211) 


\begin{tabular}{|c|c|c|c|c|}
\hline \multicolumn{2}{|c|}{ Männer } & \multicolumn{2}{|c|}{ Frauen } & \multirow[t]{2}{*}{ Autor } \\
\hline$n^{\circ}$ & $\mathrm{M}$ & $\mathrm{n}$ & $\mathbf{M}$ & \\
\hline 100 & 1652 & & & Bijlmer $(1939,136-137)$ \\
\hline 48 & 1654.5 & & & Humphreys (1926) \\
\hline 17 & 1655.0 & & & Schlaginhaufen \\
\hline 19 & 1656.3 & & & Chalmers $(1898,337)$ \\
\hline 14 & 1658 & & & Seligmann $(1909,264)$ \\
\hline 17 & 1659 & & & Friederici $(1912,323)$ \\
\hline 15 & 1664 & & & Seligmann $(1909,318)$ \\
\hline 250 & 1664 & 65 & 1566 & F. Sarasin $(1916-22,17)$ \\
\hline 31 & 1668 & 15 & 1558 & $\Rightarrow \quad(1916-22,22)$ \\
\hline 50 & 1670 & & & Bourgarel $(1865,383)$ \\
\hline 16 & 1672 & & . & $\begin{array}{l}\text { v. Luschan }(1897,243) \text { und } \\
\text { Schlaginhaufen }\end{array}$ \\
\hline & 1672 & & 1567 & $\operatorname{Koch}(1906,203-211)$ \\
\hline 35 & 1673 & & & Speiser $(1928,94)$ \\
\hline 17 & 1677 & & & Chalmers $(1898,339)$ \\
\hline 91 & 1677 & 39 & 1565 & F. Sarasin $(1916-22,21)$ \\
\hline 29 & 1678 & 12 & 1581 & $\eta \quad(1916-22,22)$ \\
\hline 12 & 1679 & 9 & 1576 & Seligmann $(1909,322-23)$ \\
\hline 65 & 1683 & & & Ollivier $(1951,190)$ \\
\hline 31 & 1684 & 12 & 1557 & F. Sarasin $(1916-22,22)$ \\
\hline 20 & 1685 & & & Ollivier $(1951,192)$ \\
\hline 21 & 1691 & & & Seligmann $(1909,263)$ \\
\hline 35 & 1693 & & & Ollivier $(1951,190)$ \\
\hline 15 & 1703 & & & $\Rightarrow \quad(1951,192)$ \\
\hline 12 & 1714 & & & Seligmann $(1909,258)$ \\
\hline 12 & 1732.1 & & & Chalmers $(1898,340)$ \\
\hline
\end{tabular}

B.N.G = Britisch-Neu-Guinea

D.N.G. = Ehemaliges Deutsch-Neu-Guinea

H.N.G. = Holländisch-Neu-Guinea

N. Hebr. = Neu-Hebriden
kursiv $=$ Neu-Irland und vorgelagerte
Inseln

Scheiden wir die neuirländischen Gruppen aus der Tabelle aus, so bleiben noch 92 Gruppen. Die Verteilung ihrer Mittelwerte auf die Kategorien der Körpergröße ist so, daß 6 Gruppen resp. $6.52 \%$ auf die sehr Kleinen, 32 oder $34.78 \%$ auf die Kleinen, 25 oder $27.17 \%$ auf die Untermittelgroßen, 13 oder $14.13 \%$ je auf die Mirtelgroßen und die Übermittelgroßen und 3 oder $3.26 \%$ auf die Großen cntfallen. Unsere Statistik über die Kategorienbesetzung bei den Neuirländern (Tabelle 1) ergab $39.6 \%$ Kleine und $27.3 \%$ Untermittelgroße.

So bietet hinsichtlich der Variabilität der Körpergröße Neuirland ein ähnliches Bild dar, wie Melanesien als Gesamtheit es tut, immerhin aber mit der Besonderheit, daß die Kleinen stärker vertreten sind. Auch distanzieren sich die größten und die kleinsten Gruppenmittelwerte der Neuirländer ziemlich stark von den extremen Mittelwerten der übrigen Gruppen Melanesiens. Tiefer als der niedrigste neuirländische Mittelwert ( $\operatorname{Lir} 1578 \mathrm{~mm}$ ) liegen mit Ausnahme zweier neubritannischer Mittelwerte (Tumuip $1556 \mathrm{~mm}$ und Baining $1572 \mathrm{~mm}$ ) und je eines. Mittelwertes von Bougainville (Nasioi $1576.9 \mathrm{~mm}$ ), West-Santo (1568 $\mathrm{mm}$ ) und Südwest-Santo (1545 mm) ausschließlich Mittelwerte von Neuguinea (24 Gruppen). Über den größten neuirländischen Mittelwert (Kambotoros-Lamassa $1659 \mathrm{~mm}$ ) hinaus gehen zehn Mittelwerte von Neu-Caledonien und den Loyalty-Inseln, sieben aus Neu-Guinea und je einer aus den Neu-Hebriden und NeuBritannien. Grosso modo ist der Pol der Kleinwüchsigen in Neu-Guinea, der Pol der Großgewachsenen im Südosten Melanesiens.

Die Eingeborenen Neuirlands und der vorgelagerten Inseln erscheinen innerhalb der Variationsbreite der gesamten Menschheit klein bis untermittelgroß; innerhalb der Schwankungsbreite Melanesiens betrachtet aber zeigen sie ein mittleres Verhalten. 


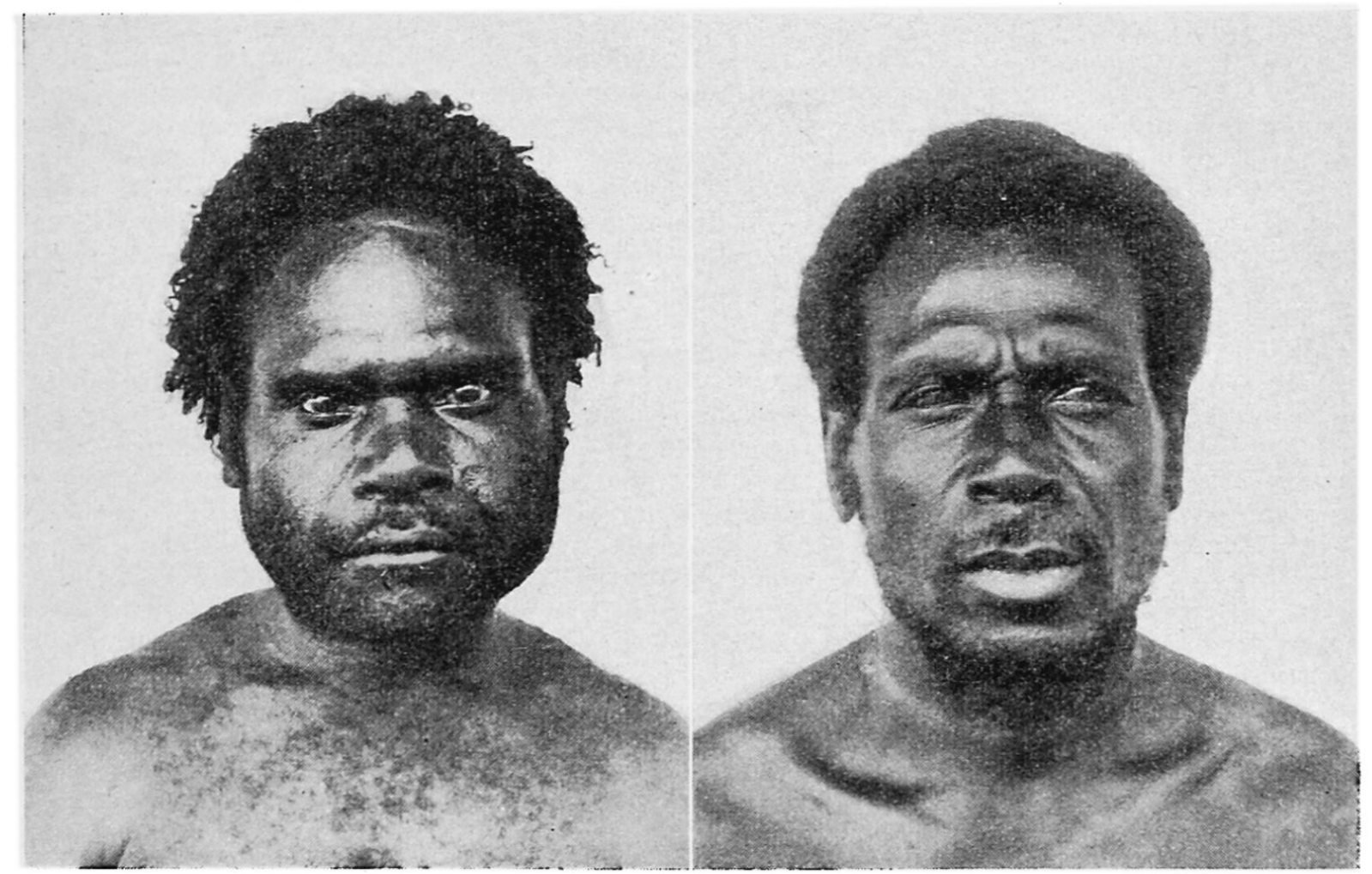

Fig. 3 Butam-Mann aus den Bergen des südlichen Neuirland (Körpergröße $M=1580.8 \mathrm{~mm}$ )
Fig. 4 Muliama-Mann von der Ostküste des südl. Neuirland (Körpergröße $M=1613.7 \mathrm{~mm}$ )

\section{LITERATUR}

1. BJJLmer, H. J. T.: Anthropological results of the Dutch Scientific Central New-Guinea Expedition $A^{0} 1920$ followed by an essay on the anthropology of the Papuans. Acad. Proefschr. Univ. Amsterdam. Leiden, 1922. 95 S.;- 2. Tapiro Pygmies and Pania Mountain-Papuans. Results of the Anthropological Mimika-Expedition in New-Guinea 1935-36. Nova Guinea, N.S. vol. III, p. 113184, E. J. Brill, Leiden, 1939. 3. Bos, HeNdrick Cornelis: Bijdrage tot de anthropologie van de bevolking der Schouten-Eilanden (Nederlandsch Nieuw-Guinee). Acad. Proefschr. Univ. Amsterdam. 1935. 186 S. 4. Bosshart, Hedwig: Anthropologische Untersuchungen im Engstligen- und Frutigtal. Arch. Jul. Klaus-Stiftung, Bd. 14, 1939, S. 1-194. 5. Bourgarel, A.: Des races de l'Océanie française. Mém. Soc. d'Anthrop., t. II, 1865, p. 375-416. 6. BRoEK, A. J. P. VAN DEM: Über Pygmäen in Niederländisch Süd-Neu-Guinea. Zeitschrift f. Ethnologie, 45. Jg., 1913. S. 23-44; - Zur Anthropologie des Bergstammes Pesechem im Innern von Niederländisch-Neu-Guinea. Nova Guinea, Vol. 7, Livr. 2, 1915, p. 233-276. Leiden, E. J. Brill. 7. Chalmers, JaMes: Anthropometrical observations on some natives of the Papuan Gulf. Journ. Anthrop. Inst., vol. 27, 1898, p. 335-342. 8. DeNiker, J.: Les indigènes de Lifou (iles Loyauté). Bull. Soc. d'Anthr., Paris, t. IV, 1893. p. 791-804. 9. FrIEDERICI, GEORG : Beiträge zur Völker- und Sprachenkunde von Deutsch-Neuguinea. MitŁeil. aus den Deutsch. Schutzgeb., Erg.h. Nr. 5, 1912. 10. FrizzI, ERsst : Kurze Vorbemerkungen über meine Reise in Bougainville und Buka. Korr.blatt d. Dtsch. Anthr. Ges., 43. Jg., 1912, S. 101-105. 11. GupPY, H. B.: The Solomon Islands and their natives. London, Swan Sonnenschein, Lowrey \& Co., 1887. 12. Haddon, A.C.: The pygmy question, in: Wollaston, Pygmies and Papuans. London, 1912, p. 303-321. 13. Hambruch, Paul: Beiträge zur Somatologie von Madagaskar, Indonesien, Bis-

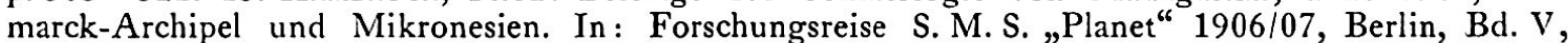
1909, S. 31-55. 14. HaGEN, B.: Anthropologischer Atlas ostasiatischer und melanesischer VölkerWiesbaden, 1898. 15. HumphreYs, C. B.: The Southern New Hebrides. An ethnological record. Cambridge, 1926. 16. Keysser, in: Neuhauss, R.: Deutsch Neu-Guinea. Berlin, Dietrich Reimer. Bd. 1, 1911, S. 494-497. 17. Kirschbaum: Ein neu entdeckter Zwergstamm auf Neu-GuineaAnthropos, 1927, S. 202. 18. Косн, J. W. R.: Beitrag zur Kenntnis der Anthropologie der Bewohner von Niederländisch-Neu-Guinea (Südliche Küste). Petrus Camper, 4e Deel, 1907, S. 202-214. 19. Lindsay Taylor, J.: Purari tribes. Im Anhang zu: Chinsery, Mountain Tribes of the Mandated Territory of New Guinea from Mt. Chapman to Mt. Hagen. Man, vol. 34, 1934, p. 113-121. 20. Luschan, F. von: Völkerkunde, in : Deutschland und seine Kolonien im Jahre 1896. Berlin, Dietr. Reimer, 1897, S. 203-269. 21. MarTin, Rudolf : Lehrbuch der Anthropologie in systematischer Darstellung. 2. Aufl., 3 Bde., Jena, Gustav Fischer, 1928. 22. Mfyer, A. B.: Anthropologische Mit- 
teilungen über die Papuas von Neu-Guinea. Mitteil. Anthrop. Ges. Wien, Bd. 4, 1874, S. 87-110. 23. Moyne and Haddon: The Pygmies of the Aiome-Mountains. Journ. R. Anthr. Inst., vol. 46, 1936. 24. Ollivier, Georges: Documents anthropologiques sur les Melanésiens orientaux. Journ. Soc. des Océanistes, t. 7, No 7, 1951, p. 187-211. 25. PoEch, Rudolf : Fälle von Zwergwuchs unter den Kai (Deutsch Neu-Guinea). Sitzgs.ber. Anthrop. Ges. Wien, Jg. 1904/05, S. [40-41]; 26. - Zwergvölker und Zwergwuchs. Mitteil. K.K. Geogr. Ges. Wien, Heft 5 \& 6, 1912. 27. RıBвE: Zwei Jahre unter den Kannibalen der Salomo-Inseln. Dresden, 1903. 28. SANDE, van DER: Ethnography and Anthropology, in: Nova Guinea, vol. 3, 1907, Leiden, Brill. 29. SARASIN, FRITZ: Anthropologie der Neu-Caledonier und Loyalty-Insulaner. Berlin, C. W. Kreidels Verl., 1916 - 1922. 30. Schellong, O.: Beiträge zur Anthropologie der Papuas. Ztschr. f. Ethnol., Bd. 23, 1891, S. 156 -230. 31. Schlaginhaufen, Otto: Ein anthropologischer Querschnitt im Südosten von Neu-Mecklenburg. Korr.bl. Dtsch. Anthr. Ges., 41. Jg., 1910, S. 109-113; 32. — Über die Pygmäenfrage in Neu-Guinea. In: Universität Zürich, Festgabe zur Einweihung der Neubauten, 18. April 1914, phil. Fak. II, 1914, S. 181-201. Schultheß \& Co., Zürich; 33. - Pygmäen in Melanesien. Arch. Suisses d'Anthrop. générale, T. 1, 1914, p. 37-42; 34. - Anthropometrische Untersuchungen an Eingeborenen in Deutsch-Neu-Guinea. Abhandl. u. Ber. Zool.-Anthrop.-Ethnogr. Mus. Dresden, Bd. 48, 1914, S. 209-219; 35. - Anthropologia Helvetica. Arch. Jul. Klaus-Stiftg., Erg.-Bd. zu Bd. 21, 1946; 36. - Zur Anthropologie der Admiralty-Inseln in Melanesien. Bull. Schweiz. Ges. Anthr. u. Ethn., 26. Jg., 1949/50, S.12-23. 37. Seligman, C. G.: A classification of the natives of British New Guinea. Journ. R. Anthrop. Inst., vol. 39, p. 246-276, 314-333. 38. Speiser, Felix : Anthropologische Messungen aus den St. Cruz-Inseln. Arch. für Anthropol., N. F. Bd. 19, 1923, S. 89-146; 39. - Anthropologische Messungen aus Espiritu Santo (Neue Hebriden). Ein Beitrag zur Pygmäenfrage. Verh. Nat. Ges: Basel, Bd. 39, 1928, S. 79-166. 40. STEPHAN, EMIL und GræBNER Fritz: Neu-Mecklenburg (Bismarck-Archipel). Berlin, Dietrich Reimer, 1907. Im Anhang: Anthropologische Messungen. bearbeitet von Schlaginhaufen. 41. Williamson, Robert W.: The Mafulu mountain people of British New Guinea. With an introductory by A. C. Haddon. London, 1912. 42. Wirz, P.: Zur Anthropologie der Biaker, Nufuresen und der Bewohner des Hinterlands der Doreh-Bai. Arch. f. Anthrop., N. F. Bd. 20, 1925, S. 185-215.

\section{LES VARIATIONS, LA RÉPARTITION GÉOGRAPHIQUE ET LA POSITION DU CONCEPT TAILLE CHEZ LES INDIGÈNES DE LA NOUVELLE-IRLANDE}

L'auteur, qui, pendant son séjour en Mélanésie de 1907 à 1909, a mené des enquêtes anthropologiques, a choisi parmi les résultats de ses recherches ce qui concerne la taille des indigènes de la Nouvelle-Irlande et iles voisines. Il a constaté une moyenne de $1610.3 \mathrm{~mm}$ pour les hommes et de 1502.5 pour les femmes et il étudie les variations de ce caractère somatique : les mensurations ayant porté sur la plus grande partie de l'île et sur 23 tribus différentes, il en résulte la possibilité d'étudier la répartition géographique des différentes tailles. La Nouvelle-Irlande a des habitants de faible taille, mais pas des pygmées. L'auteur rappelle les moyennes connues pour les Mélanésiens, qui varient, pour les hommes, entre $1384 \mathrm{~mm}$ chez les Ramu de la Nouvelle-Guinée ci-devant allemande et $1772 \mathrm{~mm}$ chez les Toaripi de la Nouvelle-Guinée britannique. Par rapport aux autres groupes humains, les indigènes de la Nouvelle-Irlande sont petits, en tout cas inférieurs à la moyenne; par rapport aux autres Mélanésiens, ils sont de grandeur moyenne.

\section{VARIABILITA, DISTRIBUZIONE GEOGRAFICA E POSIZIONE DELLA STATURA DEGLI INDIGENI DELLA NUOVA IRLANDA}

Dai risultati delle ricerche antropologiche fatte nella Melanesia durante un soggiorno che va dal 1907 al 1909, l'autore presenta il capitolo che concerne la statura degli indigeni della Nuova Irlanda. Il valore medio per gli uomini è di $1610,3 \mathrm{~mm}$ e di 1502,5 per le donne. Ne discute la variabilità. Siccome le misurazioni vennero estese alla maggior parte dell'isola ed effettuate su 23 tribù, egli riesce a fissarne la distribuzione geografica. Per la Nuova Irlanda risulta dimostrata l'esistenza di gruppi etnici di piccola statura, ma non quella di pigmei. I valori medi dei gruppi etnici della Melanesia, noti all'autore ed ordinati in un elenco, rivelano un minimo (per i maschi) di $1384 \mathrm{~mm}$ per la gente Ramu nell'ex Nuova Guinea Tedesca e un massimo di $1772 \mathrm{~mm}$ per i Toaripi nella Nuova Guinea Britannica. Nel quadro di variazione di tutta l'umanità, gli indigeni della Nuova Irlanda appaiono piccoli fino piccoli-medi (di men che media statura) : nel quadro di variazione di tutta la Melanesia essi rivelano un comportamento medio. 